\title{
Experimental and numerical investigation of an axisymmetric supersonic jet
}

\author{
By B. MATÉ ${ }^{1}$, I. A. GRAUR ${ }^{2}$, T. ELIZAROVA ${ }^{2}$, \\ I. CHIROKOV ${ }^{2}$, G. TEJEDA ${ }^{1}$, J. M. FER NÁNDEZ \\ AND S. MONTERO ${ }^{1}$ \\ ${ }^{1}$ Instituto de Estructura de la Materia, CSIC, Serrano 121, Madrid 28006, Spain \\ ${ }^{2}$ Institute for Mathematical Modeling, Russian Academy of Science, \\ Miusskaya Square, 4a, Moscow 125047, Russia
}

(Received 22 March 2000 and in revised form 3 August 2000)

A comprehensive study of a steady axisymmetric supersonic jet of $\mathrm{CO}_{2}$, including experiment, theory, and numerical calculation, is presented. The experimental part, based on high-sensitivity Raman spectroscopy mapping, provides absolute density and rotational temperature maps covering the significant regions of the jet: the zone of silence, barrel shock, Mach disk, and subsonic region beyond the Mach disk. The interpretation is based on the quasi-gasdynamic (QGD) system of equations, and its generalization (QGDR) considering the translational-rotational breakdown of thermal equilibrium. QGD and QGDR systems of equations are solved numerically in terms of a finite-difference algorithm with the steady state attained as the limit of a time-evolving process. Numerical results show a good global agreement with experiment, and provide information on those quantities not measured in the experiment, like velocity field, Mach numbers, and pressures. According to the calculation the subsonic part of the jet, downstream of the Mach disk, encloses a low-velocity recirculation vortex ring.

\section{Introduction}

The structure and the dynamical properties of supersonic jet plumes produced by the expansion of gases through an axisymmetric nozzle are well known at a qualitative level. Schlieren pictures have established the relative dimensions of the quasi-universal spatial structure of axisymmetric supersonic jets formed by the zone of silence, barrel shock, Mach disk, and slip region downstream the Mach disk (Bier \& Schmidt 1961). Impact pressure measurements in jets generated in supersonic wind tunnels have provided preliminary quantitative information (Ashkenas \& Sherman 1964), later extended to the measurements of temperatures by means of the electron beam method (Muntz, Hamel \& Maguire 1970). Pioneer work on Raman spectroscopy (Silvera \& Tommasini 1976; Luijks, Stolte \& Reuss 1981) has provided on-axis quantitative information about densities and rotational temperatures in the zone of silence of the jet. The possibility of a complete density and thermal mapping of the jet by means of Raman spectroscopy has been shown recently (Tejeda et al. 1996).

A first semi-empirical quantification of density and temperature in the zone of silence of the jet, based on the isentropic calculation by the method of characteristics, has been given in terms of the local Mach number (Ashkenas \& Sherman 1964; Murphy \& Miller 1984; Miller 1988). The dynamics of normal shock waves, similar 
to the Mach disk of supersonic jets, has been widely studied in one dimension, theoretically (Mott-Smith 1951), numerically (Koura 1997) using the direct simulation Monte Carlo method (DSMC) developed by Bird (1994), and experimentally (Robben \& Talbot 1966a,b; Alsmeyer 1976; Pham-Van-Diep, Erwin \& Muntz 1989).

Theoretical and numerical studies of the global jet are however scarce due to the wide range of flow conditions from the continuum, near the nozzle, to the molecular regime, at large distances downstream. Further, the breakdown of thermodynamical equilibrium among the vibrational, rotational, and translational degrees of freedom, specially at the shock regions, poses an additional computational challenge. In an attempt to overcome such difficulties a multizone approach combining Navier-Stokes equations, near the nozzle, with a DSMC calculation far away from it, has been proposed recently by Ivanov et al. (1998).

In the present paper we report a detailed quantitative experimental investigation of a reference supersonic jet of $\mathrm{CO}_{2}$ by means of high-sensitivity Raman spectroscopy, jointly with its simulation within a two-dimensional computational approach based on the numerical solution of the quasi-gasdynamic (QGD) equations developed by Elizarova et al. (1995).

\section{Experimental}

The experimental part of present work has been carried out in the miniature jet diagnostic facility commissioned at the Instituto de Estructura de la Materia, CSIC, Madrid (Maté 1997; Montero et al. 2000). It consists of a continuous flow expansion chamber with diagnostics based on linear Raman spectroscopy. The corresponding spectra were recorded with a very high-sensitivity instrument (Tejeda, FernándezSánchez \& Montero 1997) particularly suited for rarefied gas studies. In this facility, fields of up to $6 \mathrm{~mm}$ across, and $25 \mathrm{~mm}$ along the flow, can be monitored with spatial resolution of few $\mu \mathrm{m}$. At present, the quantities that can be measured at any point of the flow field are the absolute number density of molecules, within a range of about six orders of magnitude, the rotational temperature, and the degree of condensation (Maté, Tejeda \& Montero 1998; Montero et al. 2000).

The reference stationary jet of $\mathrm{CO}_{2}$ investigated here was generated through a Campargue-type axisymmetric nozzle (Campargue 1984) of radius $R_{e}=156.5 \mu \mathrm{m}$, with channel length of about $1 \mathrm{~mm}$. The gas was expanded into the sample chamber at a stagnation pressure $p_{0}=203 \mathrm{kPa}$, and temperature $T_{0}=300 \mathrm{~K}$. Under these conditions the residual pressure attained in the expansion chamber by means of the current vacuum pumps $-\mathrm{a} 400 \mathrm{~m}^{3} \mathrm{~h}^{-1}$ Roots pump backed by a rotary pump of $70 \mathrm{~m}^{3} \mathrm{~h}^{-1}$ - becomes stabilized at about $40 \mathrm{~Pa}$.

In order to maintain a stationary normal shock wave (Mach disk) approximately centred in the observable domain, the residual pressure was increased up to $p_{\infty}=81 \mathrm{~Pa}$ by inlet of atmospheric air through a needle valve. According to the empirical relation (Bier \& Schmidt 1961; Ashkenas \& Sherman 1964)

$$
z_{M}=1.34 R_{e}\left(p_{0} / p_{\infty}\right)^{1 / 2},
$$

a Mach disk is predicted at $z_{M} \approx 66 R_{e}$ from the nozzle. Here it is observed at $z_{M}=64 R_{e}$. The background gas of the reference expansion of $\mathrm{CO}_{2}$ is an approximately $1: 1$ mixture of $\mathrm{CO}_{2}$ and air, a fact not to be ignored when comparing the present experimental results with the numerical calculations based on the single molecular species $\mathrm{CO}_{2}$, specially in the region beyond the barrel shock.

Also, a factor to be taken into account when comparing experimental and numerical 


\begin{tabular}{lll}
\hline & \multicolumn{1}{c}{ QGD } & \multicolumn{1}{c}{ QGDR } \\
$\gamma$ & 1.35 & 1.40 \\
$T_{e}$ & $254.6 \mathrm{~K}$ & $249.2 \mathrm{~K}$ \\
$p_{e}$ & $107499 \mathrm{~Pa}$ & $105751 \mathrm{~Pa}$ \\
$n_{e}$ & $3.058 \times 10^{25} \mathrm{~m}^{-3}$ & $3.074 \times 10^{25} \mathrm{~m}^{-3}$ \\
$\rho_{e}$ & $2.234 \mathrm{Kg} \mathrm{m}^{-3}$ & $2.246 \mathrm{Kg} \mathrm{m}^{-3}$ \\
$u_{e}$ & $257.4 \mathrm{~m} \mathrm{~s}^{-1}$ & $259.3 \mathrm{~m} \mathrm{~s}^{-1}$ \\
$p_{\infty} / p_{e}$ & $0.7536 \times 10^{-3}$ & $0.7660 \times 10^{-3}$ \\
$\lambda_{e}$ & $2.330 \times 10^{-8} \mathrm{~m}$ & $2.318 \times 10^{-8} \mathrm{~m}$ \\
$K n_{e}$ & $7.444 \times 10^{-5}$ & $7.406 \times 10^{-5}$
\end{tabular}

TABLE 1. Nozzle exit quantities of the $\mathrm{CO}_{2}$ jet in the QGD and QGDR runs from source conditions $R_{e}=156.5 \mu \mathrm{m}, p_{0}=203 \mathrm{kPa}, T_{0}=300 \mathrm{~K}, n_{0}=4.49 \times 10^{25} \mathrm{~m}^{-3}$, assuming $M a_{e}=1.01$.

results is the condensation produced in the jet. Actual rotational temperatures depend on condensation, but the present numerical simulation does not take into account this effect. Thus, to be comparable, experimental rotational temperatures need to be corrected for condensation. As has been shown recently (Maté et al. 1998), from the analysis of the evolution of experimental density number, rotational temperature, and vibrational temperature of several supersonic jets of $\mathrm{CO}_{2}$, performed in terms of energy and momentum conservation, relaxation time, and intermolecular binding energy, the condensed gas fraction at each point of the zone of silence can be deduced. The conversion of the energy released by condensation into rotational temperature enhancement is immediate. For the present expansion it has been estimated that about $9 \%$ of the expanded gas becomes condensed, with a noticeable increase of the observed rotational temperatures in the zone of silence.

In the present work we report the first experimental high-resolution maps of absolute density and rotational temperature of a supersonic jet. These maps have been generated from the Raman spectroscopic data, according to the procedure described by Tejeda et al. (1996). Thereafter several instrumental improvements have been introduced, namely, (i) a faster and more accurate procedure to record and process the Raman spectra, and (ii) a far better positioning accuracy to define the points $P(r, z)$ of the jet where the spectra are recorded. The measurement points $P(r, z)$, covering a flow field $0 \leqslant r \leqslant 40 R_{e}$ and $2 R_{e} \leqslant z \leqslant 155 R_{e}$, can now be determined with an accuracy of $\pm 1 \mu \mathrm{m}$. The effective spatial resolution is $\Delta r \simeq 0.16 R_{e}$, and $\Delta z \simeq 0.06 R_{e}$, centred at any point $P(r, z)$ of the flow field.

The density map shown in figure $1(a)$ was generated from the Raman intensity of the $v=1388.2 \mathrm{~cm}^{-1}$ vibrational band of $\mathrm{CO}_{2}$ recorded in a grid of 2200 points $P(r, z)$ of the jet covering the flow field. The reported absolute densities are probably accurate to $10 \%$. The map of figure $1(c)$ was generated from the rotational temperatures measured from 2200 pure rotational spectra, one per $P(r, z)$ point. Rotational temperatures are estimated to be accurate to $5 \%$, excluding points within the Mach disk and barrel shock, where a non-Boltzmann distribution has been observed. At these points the temperatures of the map correspond to the best linear fit to a Boltzmann plot.

All the characteristic features of axisymmetric jets (Miller 1988; Abramovich 1991) can be identified in the density and temperature maps of figure $1(a$ and $c)$ : the zone of silence, barrel shock, Mach disk, the interference between these two shocks, and the slip region downstream of the Mach disk. The present data cover a wide range of Mach numbers, approximately $1 \leqslant M a \leqslant 11$ in the zone of silence, with 

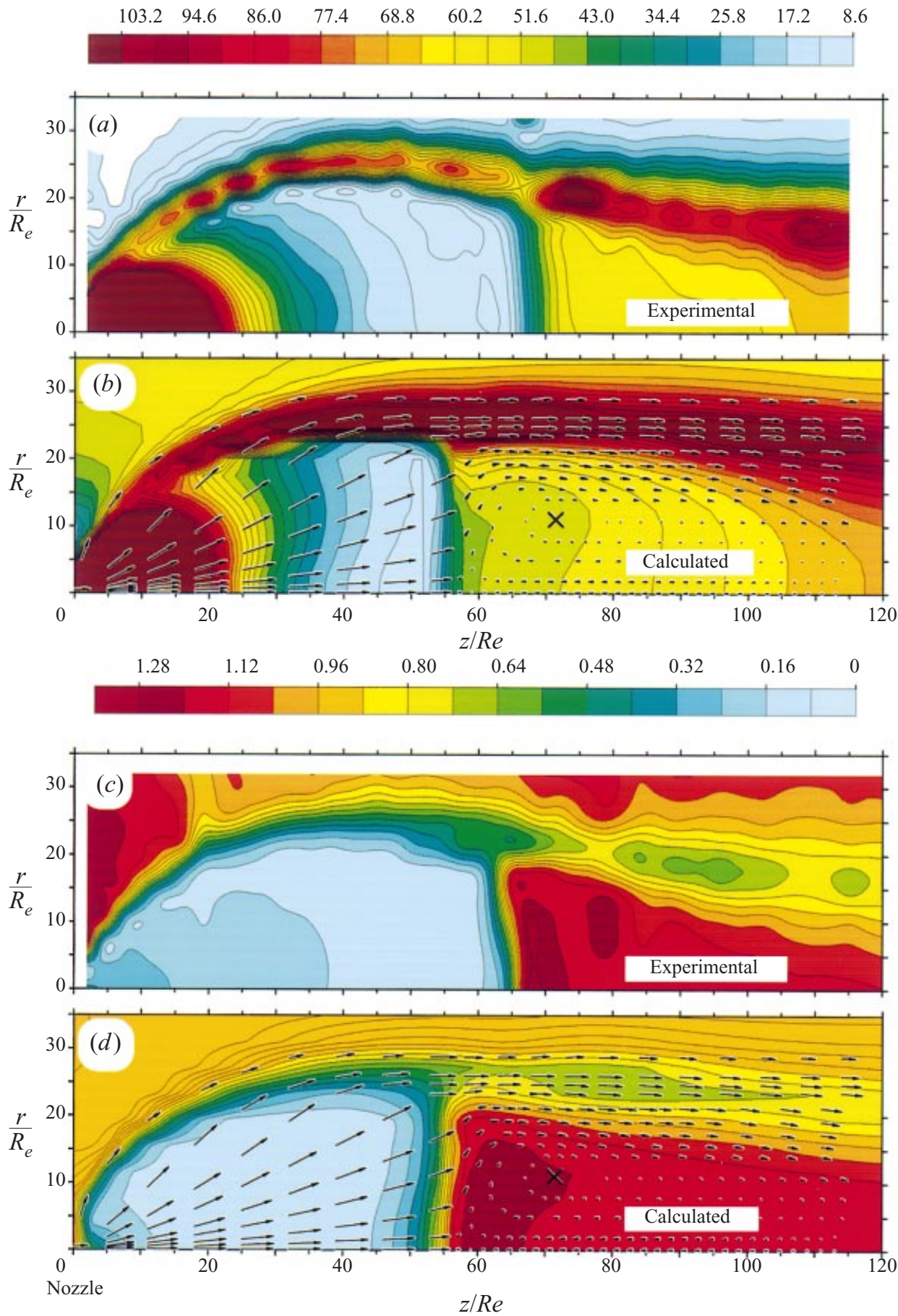

FIGURE 1. Supersonic jet of $\mathrm{CO}_{2}$ for the source and nozzle exit conditions of table 1. (a) Experimental number density $\left(10^{5} \times\left(n / n_{e}\right)\right)$, (b) QGDR calculated number density and velocity, $(c)$ experimental rotational temperature $\left(T_{r o t} / T_{e}\right),(d)$ QGDR calculated rotational temperature and velocity. Largest calculated velocity is $u_{\max }=2.37 u_{e}=615 \mathrm{~m} \mathrm{~s}^{-1}$. The vortex is located at ' $X$ '. 
$M a<1$ downstream of the Mach disk; the Knudsen number for the mean free path $\lambda$ ranges from $K n_{e}=\lambda_{e} / 2 R_{e}=7.4 \times 10^{-5}$, at the nozzle exit, to a local value $K n=(\lambda / \rho) /(\mathrm{d} \rho / \mathrm{d} z)=0.21$ (variable hard-sphere approximation) within the Mach disk, close to the limit of applicability of Navier-Stokes equations (Bird 1994). Quantitative in nature, the reported data provide suitable material for the validation of theories and numerical procedures, as shown below.

\section{Theory}

The axisymmetric jet configuration described above is studied numerically in present work on the basis of the quasi-gasdynamic (QGD) system of equations developed by Elizarova et al. (1995). This system, a novel approach for the description of viscous gas flows, has already been applied to several problems: the flow around a semi-infinite sharp flat plate parallel to a free stream (Elizarova et al. 1995), the flow around an infinitely thin circular disk perpendicular to a supersonic flow (Lengrand et al. 1995; Elizarova et al. 1997), the shock wave structure (Graur, Elizarova \& Lengrand 1997; Elizarova, Lengrand \& Graur 1999), and the shock wave configurations in underexpanded jets for regular and Mach reflections (Graur, Elizarova \& Lengrand 1999). However, a complete theoretical-experimental study using QGD equations is presented here for the first time.

Comparison with Navier-Stokes and DSMC simulations shows that the QGD equations can be used efficiently for the simulation of complex gasdynamical problems. QGD equations appear to have advantages compared to Navier-Stokes equations at large Mach numbers, for instance in shock wave problems for $M a \geqslant 5$, and are less sensitive to computational instabilities induced by strong flow gradients.

In order to account for the breakdown of translational-rotational thermal equilibrium the QGD equations have been extended to the QGDR equations by Chirokov, Elizarova \& Lengrand (1999). The present experimental data include a wealth of information about rotational temperatures explored here in terms of the QGDR generalization.

\subsection{Quasi-gasdynamic equations and problem formulation}

A gasdynamic system consists of three differential equations accounting for $(a)$ conservation of mass (continuity equation)

$$
\frac{\partial \rho}{\partial t}+\nabla_{i} J^{i}=0
$$

(b) conservation of momentum

$$
\frac{\partial\left(\rho u^{k}\right)}{\partial t}+\nabla_{i} J^{i} u^{k}+\nabla^{k} p=\nabla_{i} \Pi^{i k},
$$

and $(c)$ conservation of total energy

$$
\frac{\partial E}{\partial t}+\nabla_{i} \frac{J^{i}}{\rho}(E+p)+\nabla_{i} q^{i}=\nabla_{i}\left(\Pi^{i k} u^{k}\right) .
$$

To close the system (2)-(4) the mass flux vector $J^{i}$, the shear-stress tensor $\Pi^{i k}$, and the heat flux vector $q^{i}$ must be expressed as a function of the macroscopic flow quantities of density $\rho$, velocity coordinates $u_{i}$, and pressure $p$.

Different choices for $J^{i}, \Pi^{i k}, q^{i}$ lead either to the Navier-Stokes equations, or to 
the present QGD equations. The Navier-Stokes equations are derived from

$$
\begin{gathered}
J^{i}=\rho u^{i}, \\
\Pi^{i k}=\Pi_{N S}^{i k}=\mu\left[\nabla^{k} u^{i}+\nabla^{i} u^{k}-(2 / 3) g^{i k} \nabla_{j} u^{j}\right]+\zeta g^{i k} \nabla_{j} u^{j},
\end{gathered}
$$

and

$$
q^{i}=-\kappa \nabla^{i} T,
$$

where $g^{i k}$ is the metric tensor, $\mu$ and $\kappa$ the viscosity and heat conductivity coefficients, and $\zeta$ the bubble viscosity. The gasdynamic variables $\rho, u_{i}$, and $p$ involved here are instantaneous space-averaged quantities, and $\Pi_{N S}^{i k}$ is the Navier-Stokes shear-stress tensor.

In contrast to the Navier-Stokes equations, if the gasdynamic quantities $\rho, u_{i}$, and $p$ are defined by means of time-space averaging-instead of space averaging-the system (2)-(4) can be closed by two other ways, in particular by

$$
\begin{gathered}
J^{i}=\rho\left(u^{i}-\frac{\tau}{\rho}\left[\nabla_{j}\left(\rho u^{i} u^{j}\right)+\nabla^{i} p\right]\right), \\
\Pi^{i k}=\Pi_{N S}^{i k}+\tau u^{i}\left[\rho u^{j} \nabla_{j} u^{k}+\nabla^{k} p\right]+\tau g^{i k}\left[u_{j} \nabla^{j} p+\gamma p \nabla_{j} u^{j}\right],
\end{gathered}
$$

and

$$
q^{i}=-\kappa \nabla^{i} T-\tau \rho u^{i}\left[u^{j} \nabla_{j} \varepsilon+p u_{j} \nabla^{j}(1 / \rho)\right],
$$

where $\tau=\mu / \rho$ is the Maxwellian relaxation time, and $\varepsilon=p /(\rho(\gamma-1))$. Equations (2)-(4), with (8)-(10), are the QGD equations, a system where mass, momentum, total energy, and entropy conservation laws are valid for the moving volume (Elizarova et al. 1995, 1997; Sheretov 1997, 1999). They can also be derived by moment averaging over the velocity space $v_{i}$ of the model kinetic equation

$$
\frac{\partial f}{\partial t}+\nabla_{i} v^{i} f^{(0)}=\nabla_{i} \tau \nabla_{j} v^{i} v^{j} f^{(0)}+\frac{f^{(0)}-f}{\tau},
$$

where $f=f\left(x_{i}, v_{i}, t\right)$ is the one-particle distribution function, and $f^{(0)}$ the corresponding local-Maxwellian distribution function. The macroscopic equations obtained must then be generalized for gases with $\gamma \neq 5 / 3$ and Prandtl number $\operatorname{Pr} \neq 1$ (Elizarova \& Chetverushkin 1985; Sheretov 1997; Elizarova et al. 1999).

For slightly non-equilibrium flows the time-space averaged quantities and the space-averaged quantities are similar, the QGD and Navier-Stokes systems differing by $O(\tau)$. For stationary flows the dissipative terms (terms in $\tau$ ) in the QGD equations appear as Navier-Stokes terms, the QGD and Navier-Stokes equations differing by the additional terms whose asymptotic order is $O\left(\tau^{2}\right)$ for $\tau \rightarrow 0$, or in the dimensionless form of the equations, $O\left(K n^{2}\right)$ for $K n \rightarrow 0$ (Graur et al. 1997). The boundary layer approximation for the QGD equations leads to the classic Prandtl equation system (Sheretov 1997).

The QGD equations have also been particularized for two-dimensional axisymmetric expansions (Lengrand et al. 1995). In the $(r, z)$ coordinates used in present work they read

$$
\begin{aligned}
\frac{\partial \rho}{\partial t} & +\frac{1}{r} \frac{\partial}{\partial r}\left(r \rho u_{r}\right)+\frac{\partial}{\partial z}\left(\rho u_{z}\right) \\
= & \frac{1}{r} \frac{\partial}{\partial r} \tau \frac{\partial}{\partial r}\left(r \rho u_{r}^{2}\right)+\frac{\partial}{\partial z} \tau \frac{\partial}{\partial z}\left(\rho u_{z}^{2}\right)+\frac{1}{r} \frac{\partial}{\partial r}\left(r \tau \frac{\partial p}{\partial r}\right)+\frac{\partial}{\partial z}\left(\tau \frac{\partial p}{\partial z}\right) \\
& +\frac{1}{r} \frac{\partial}{\partial r}\left(r \tau \frac{\partial}{\partial z} \rho u_{r} u_{z}\right)+\frac{\partial}{\partial z}\left(\frac{\tau}{r} \frac{\partial}{\partial r}\left(r \rho u_{r} u_{z}\right)\right),
\end{aligned}
$$




$$
\begin{aligned}
\frac{\partial \rho u_{r}}{\partial t}+ & \frac{1}{r} \frac{\partial}{\partial r}\left(r \rho u_{r}^{2}\right)+\frac{\partial}{\partial z}\left(\rho u_{r} u_{z}\right)+\frac{\partial p}{\partial r} \\
= & \frac{1}{r} \frac{\partial}{\partial r}\left(\tau \frac{\partial}{\partial r} r \rho u_{r}^{3}\right)+\frac{\partial}{\partial z}\left(\tau \frac{\partial}{\partial z} \rho u_{r} u_{z}^{2}\right)+2 \frac{1}{r} \frac{\partial}{\partial r}\left(r \tau \frac{\partial p u_{r}}{\partial r}\right) \\
& -2 p \tau \frac{u_{r}}{r^{2}}+\frac{\partial}{\partial r}\left(\frac{\tau}{r} \frac{\partial}{\partial r}\left(r p u_{r}\right)\right)+\frac{\partial}{\partial z}\left(\tau \frac{\partial p u_{r}}{\partial z}\right)+\frac{1}{r} \frac{\partial}{\partial r}\left(r \tau \frac{\partial}{\partial z}\left(\rho u_{r}^{2} u_{z}\right)\right) \\
& +\frac{\partial}{\partial z}\left(\frac{\tau}{r} \frac{\partial}{\partial r}\left(r \rho u_{r}^{2} u_{z}\right)\right)+\frac{\partial}{\partial r}\left(\tau \frac{\partial p u_{z}}{\partial z}\right)+\frac{\partial}{\partial z}\left(\tau \frac{\partial p u_{z}}{\partial r}\right),
\end{aligned}
$$

$$
\begin{aligned}
\frac{\partial \rho u_{z}}{\partial t} & +\frac{1}{r} \frac{\partial}{\partial r}\left(r \rho u_{r} u_{z}\right)+\frac{\partial}{\partial z}\left(\rho u_{z}^{2}\right)+\frac{\partial p}{\partial z} \\
= & \frac{1}{r} \frac{\partial}{\partial r}\left(\tau \frac{\partial}{\partial r} r \rho u_{r}^{2} u_{z}\right)+\frac{\partial}{\partial z}\left(\tau \frac{\partial}{\partial z} \rho u_{z}^{3}\right)+\frac{1}{r} \frac{\partial}{\partial r}\left(r \tau \frac{\partial p u_{z}}{\partial r}\right)+3 \frac{\partial}{\partial z}\left(\tau \frac{\partial p u_{z}}{\partial z}\right) \\
& +\frac{1}{r} \frac{\partial}{\partial r}\left(r \tau \frac{\partial}{\partial z} \rho u_{r} u_{z}^{2}\right)+\frac{\partial}{\partial z}\left(\frac{\tau}{r} \frac{\partial}{\partial r}\left(r \rho u_{r} u_{z}^{2}\right)\right)+\frac{1}{r} \frac{\partial}{\partial r}\left(r \tau \frac{\partial p u_{r}}{\partial z}\right)+\frac{\partial}{\partial z}\left(\frac{\tau}{r} \frac{\partial}{\partial r} r p u_{r}\right),
\end{aligned}
$$

$$
\begin{aligned}
\frac{\partial E}{\partial t}+ & \frac{1}{r} \frac{\partial}{\partial r}\left(r u_{r}(E+p)\right)+\frac{\partial}{\partial z}\left(u_{z}(E+p)\right) \\
= & \frac{1}{r} \frac{\partial}{\partial r}\left(\tau \frac{\partial}{\partial r} r u_{r}^{2}(E+2 p)\right)+\frac{\partial}{\partial z}\left(\tau \frac{\partial}{\partial z} u_{z}^{2}(E+2 p)\right) \\
& +\frac{1}{r} \frac{\partial}{\partial r}\left(r \tau \frac{\partial}{\partial r} p \frac{u_{r}^{2}}{2}\right)+\frac{\partial}{\partial z}\left(\tau \frac{\partial}{\partial z} p \frac{u_{z}^{2}}{2}\right)+\frac{1}{r} \frac{\partial}{\partial r}\left(r \tau \frac{\partial}{\partial r} p \frac{u_{z}^{2}}{2}\right)+\frac{\partial}{\partial z}\left(\tau \frac{\partial}{\partial z} \frac{p u_{r}^{2}}{2}\right) \\
& +\frac{\gamma}{\gamma-1}\left(\frac{1}{r} \frac{\partial}{\partial r}\left(\frac{\tau p}{\rho} r \frac{\partial p}{\partial r}\right)+\frac{\partial}{\partial z}\left(\frac{\tau p}{\rho} \frac{\partial p}{\partial z}\right)\right) \\
& +\frac{\gamma P r}{\gamma-1}\left(\frac{1}{r} \frac{\partial}{\partial r}\left(p \tau r \frac{\partial}{\partial r} \frac{p}{\rho}\right)+\frac{\partial}{\partial z}\left(p \tau \frac{\partial}{\partial z} \frac{p}{\rho}\right)\right) \\
& +\frac{1}{r} \frac{\partial}{\partial r}\left(\tau r \frac{\partial}{\partial z} u_{r} u_{z}(E+2 p)\right)+\frac{\partial}{\partial z}\left(\frac{\tau}{r} \frac{\partial}{\partial r} r u_{r} u_{z}(E+2 p)\right),
\end{aligned}
$$

where

$$
E=\left(\rho u_{i}^{2}\right) / 2+p /(\gamma-1)
$$

Compared to the Navier-Stokes system, the QGD system includes additional space derivatives for $p$. Thus, as has already been discussed (Elizarova et al. 1995), an additional boundary condition is required. 


\subsection{QGDR generalization}

The translational-rotational non-equilibrium in a gas with two rotational degrees of freedom can be accounted for by means of the QGDR system of equations:

$$
\begin{gathered}
\frac{\partial}{\partial t} \rho+\nabla_{i} \rho u^{i}-\nabla_{i} \tau\left(\nabla_{j} \rho u^{i} u^{j}+\nabla^{i} p_{t r}\right)=0 \\
\frac{\partial}{\partial t} \rho u^{k}+\nabla_{i} \rho u^{i} u^{k}+\nabla^{k} p_{t r}=\nabla_{i} \tau \nabla_{j} \rho u^{i} u^{j} u^{k}+\nabla_{i} \tau\left(\nabla^{i} p_{t r} u^{k}+\nabla^{k} p_{t r} u^{i}\right)+\nabla^{k} \tau \nabla_{i} p_{t r} u^{i} \\
\frac{\partial}{\partial t} E_{t r}+\nabla_{i} u^{i}\left(E_{t r}+p_{t r}\right)=\nabla_{i} \tau\left(\nabla_{j}\left(E_{t r}+2 p_{t r}\right) u^{i} u^{j}+\frac{1}{2} \nabla^{i} u_{k} u^{k} p_{t r}\right) \\
+\frac{5}{2} \nabla_{i} \tau \frac{p_{t r}}{\rho} \nabla^{i} p_{t r}+\operatorname{Pr}^{-1} \frac{5}{2} \nabla_{i} \tau p_{T} \nabla_{t r}^{i} \frac{p_{t r}}{\rho}+S_{t r}, \\
\frac{\partial}{\partial t} E_{r o t}+\nabla_{i} u^{i} E_{r o t}=\nabla_{i} \tau \nabla_{j} u^{i} u^{j} E_{r o t}+\nabla_{i} \tau \frac{p_{r o t}}{\rho} \nabla^{i} p_{t r}+\operatorname{Pr}^{-1} \nabla_{i} \tau p_{t r} \nabla^{i} \frac{p_{r o t}}{\rho}+S_{r o t} .
\end{gathered}
$$

The equations above have been derived by moment averaging of the model kinetic equation (11) replacing $f^{(0)}$ by the product of two distribution functions, respectively for the translational and rotational temperatures (Chirokov et al. 1999). The energy exchange terms

$$
S_{t r}=-S_{\text {rot }}=\frac{3}{5 \tau_{\text {rot }}}\left(p_{\text {rot }}-p_{t r}\right),
$$

in (19) and (20) involve the rotational relaxation time $\tau_{\text {rot }}$. It can be estimated as $\tau_{\text {rot }}=Z \tau_{c}$, where $\tau_{c}=\tau((7-2 \omega)(5-2 \omega)) / 30$ is the mean collisional time, and

$$
Z=\frac{Z^{\infty}}{1+\left(\pi^{3 / 2} / 2\right)\left(T^{*} / T_{t r}\right)^{1 / 2}+\left(\pi+\pi^{2} / 4\right)\left(T^{*} / T_{t r}\right)},
$$

the so-called rotational collision number (Parker 1959; Bird 1994). The parameters $\omega=0.93$ (Bird 1994), $Z^{\infty}=17.9$ and $T^{*}=100 \mathrm{~K}$ (Maté et al. 1998) have been used here for $\mathrm{CO}_{2}$. At thermal equilibrium $p_{t r}=p_{r o t}=p_{a v}=p$, and the QGDR system turns into the one-temperature QGD system with perfect-gas specific heat ratio $\gamma=7 / 5$.

Average pressure and temperature derived from the QGDR generalization, to be compared to the homologous QGD quantities, $p$ and $T$, are defined as

$$
p_{a v}=\left(3 p_{t r}+2 p_{r o t}\right) / 5=\rho(\mathscr{R} / \mathscr{M}) T_{a v},
$$

where $\mathscr{R}=8.31451 \mathrm{JK}^{-1} \mathrm{~mol}^{-1}$ is the universal gas constant, and $\mathscr{M}$ the molar mass.

\section{Numerical calculations}

\subsection{Flow and boundary conditions}

The calculations were carried out under flow conditions that allow comparison with the experimental results obtained in the miniature jet diagnostics facility described above.

For the present jet a variable specific heat ratio ranging from $\gamma=1.31$ at the nozzle exit, to $\gamma=1.36$ at $z \approx 50 R_{e}$ has been deduced from the experimental vibrational cooling (Montero et al. 2000). In the QGD calculations a fixed value $\gamma=1.35$ has been assumed along the jet, corresponding to $\xi=2.7$ excited internal degrees of 
freedom ( 2 rotational +0.7 vibrational) according to the relation (Bird 1994)

$$
\gamma=\frac{5+\xi}{3+\xi}
$$

A Prandtl number

$$
\operatorname{Pr}=\frac{4 \gamma}{(9 \gamma-5)}=0.755,
$$

estimated from Eucken's approximation (Chapman \& Cowling 1952), has been used in the QGD calculations. In turn, the QGDR model requires a perfect-gas specific heat ratio $\gamma=1.4$ for $\mathrm{CO}_{2}$, which leads to the value $\mathrm{Pr}=0.737$ used in the present QGDR calculations.

The viscosity coefficient has been treated within the variable hard-sphere (VHS) model, which leads to a thermal dependence (Chapman \& Cowling 1952; Schlichting 1955)

where

$$
\mu=\mu_{e}\left(\frac{T}{T_{e}}\right)^{\omega}
$$

$$
\mu_{e}=\mu_{r e f}\left(\frac{T_{e}}{T_{r e f}}\right)^{\omega} .
$$

The VHS molecular diameter $d_{r e f}=d\left(T_{r e f}\right)=5.62 \times 10^{-10} \mathrm{~m}$ and the parameters $\omega=0.93$, and $\mu_{\text {ref }}=\mu\left(T_{\text {ref }}\right)=1.38 \times 10^{-5} \mathrm{~N} \mathrm{~s} \mathrm{~m}^{-2}$ at $T_{\text {ref }}=273 \mathrm{~K}$ (Bird 1994), have been used here for $\mathrm{CO}_{2}$. The nozzle exit quantities, some of them required for the calculation, are reported in table 1 . They have been obtained from the source conditions of the experiment by means of the isentropic approximation, assuming $M a_{e}=u_{e} / a_{e}=1.01$.

At the nozzle exit we suppose a laminar boundary layer of width $\delta=0.18 R_{e}$. Since the walls of the nozzle are considered to be adiabatic, Crokko's integral (Schlichting 1955) has been used for the temperature distribution near the wall. So the axial velocity and temperature distributions at the nozzle exit ( $\mathrm{AB}$ boundary in figure 2) are taken as follows:

Inside the boundary layer $\left(R_{e}-\delta \leqslant r \leqslant R_{e}\right)$

$$
\frac{u_{z e}(r)}{u_{z e}}=1.5\left(\frac{R_{e}-r}{\delta}\right)-0.5\left(\frac{R_{e}-r}{\delta}\right)^{3},
$$

and

$$
\frac{T_{e}(r)}{T_{e}}=1+\frac{\gamma-1}{2} M a_{e}^{2}\left(1-\left(\frac{u_{z e}(r)}{u_{z e}}\right)^{2}\right) \sqrt{P r},
$$

valid for the adiabatic wall. The density is found from the equation of state under the assumption that the condition $(\partial p / \partial r)=0$ holds within the boundary layer (Schlichting 1955).

Outside the boundary layer $\left(0 \leqslant r<R_{e}-\delta\right)$

$$
u_{z e}(r)=u_{e}, \quad T_{e}(r)=T_{e} .
$$

For the jet expanding into the undisturbed residual gas the boundary conditions, referred to the scheme of the computational domain shown in figure 2, are as follows: $\mathrm{AB}$ boundary, at the nozzle exit

$$
u_{z}(r)=u_{z e}(r), \quad u_{r}=0, \quad p_{\sigma}(r)=p_{e}, \quad T_{\sigma}(r)=T_{e}(r) ;
$$




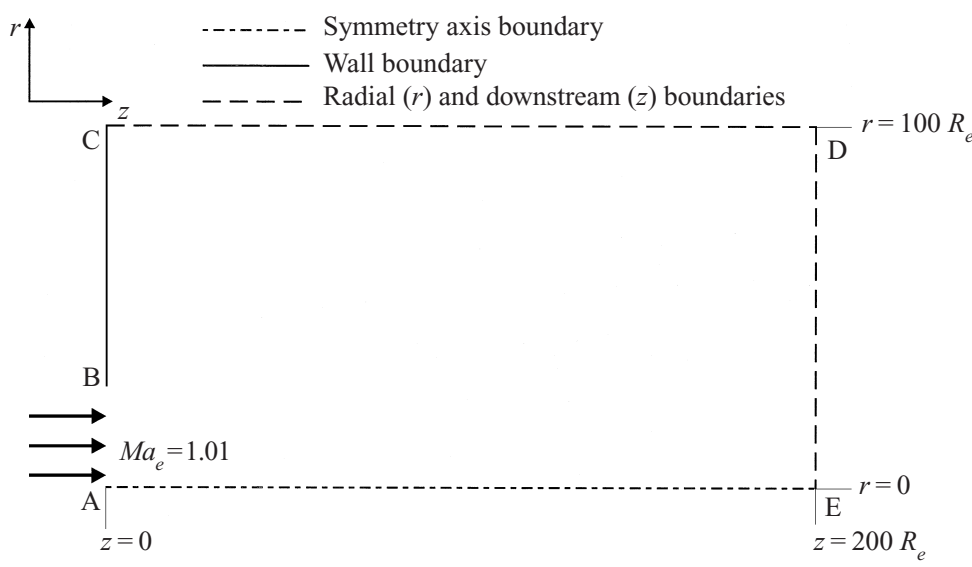

FiguRE 2. Definition of the problem and the computational domain.

$\mathrm{BC}$ boundary, on the nozzle wall

$$
u_{z}=0, \quad \frac{\partial u_{r}}{\partial z}=0, \quad \frac{\partial p_{\sigma}}{\partial z}=0, \quad \frac{\partial T_{\sigma}}{\partial z}=0 ;
$$

CD radial boundary (undisturbed flow)

$$
\frac{\partial u_{z}}{\partial r}=0, \quad u_{r}=0, \quad p_{\sigma}=p_{\infty}, \quad T_{\sigma}=T_{\infty} ;
$$

DE (downstream) boundary, where the so-called 'soft' conditions are prescribed

$$
\frac{\partial u_{z}}{\partial z}=0, \quad \frac{\partial u_{r}}{\partial z}=0, \quad \frac{\partial p_{\sigma}}{\partial z}=0, \quad \frac{\partial T_{\sigma}}{\partial z}=0 ;
$$

AE symmetry axis boundary

$$
\frac{\partial u_{z}}{\partial r}=0, \quad u_{r}=0, \quad \frac{\partial p_{\sigma}}{\partial r}=0, \quad \frac{\partial T_{\sigma}}{\partial r}=0 .
$$

Index $\sigma$, which refers equally to the QGD or QGDR models, is $\sigma=a v$ for QGD, and $\sigma=t r=r o t$ for QGDR, assumming translational-rotational thermal and pressure equilibrium at the nozzle exit (Maté et al. 1998) (AB boundary), and at the BC nozzle wall and $\mathrm{CD}$ radial boundaries.

\subsection{Numerical algorithm}

For the QGD and QGDR models the computational domain of figure 2 is covered by a rectangular grid with steps $h_{r}$ and $h_{z}$. For $r<R_{e}$ the grid in the radial direction is uniform, with the smallest spatial step $h_{r}=h_{r, \min }=0.1 R_{e}$. On the other hand, for $r>R_{e}, h_{r}$ increases between adjacent cells by a constant factor 1.05. The spatial step in the axial direction, $h_{z}=R_{e}$, is uniform. The limit of the computational domain in the radial direction, $r_{\max }=100 R_{e}$, is taken large enough for the radial CD boundary to be located entirely in the undisturbed free stream.

For computational convenience the systems of equations (12) to (15), and (17) to (20), were reduced to dimensionless form by means of the relations given by Lengrand et al. (1995). The computation was carried out according to a finite-difference scheme where the spatial derivatives are approximated with accuracy $O\left(h^{2}\right)$ (centred scheme). When solving the QGD or QGDR system of equations, oscillations appear for high Mach numbers in regions with strong gradients. To overcome this problem $\tau$ is 


\begin{tabular}{|c|c|c|c|c|}
\hline Variant & 1 & 2 & 3 & QGDR \\
\hline $\begin{array}{l}h_{r, \min } / R_{e} \\
h_{r, \max } / R_{e} \\
h_{z} / R_{e} \\
\alpha \\
\beta \\
\Delta t \\
\left(N_{z} \times N_{r}\right)_{\text {grid }} \\
\text { Iterations }\end{array}$ & $\begin{array}{l}0.1 \\
4.8 \\
1 \\
0.005 \\
0.2 \\
1.12 \\
201 \times 92 \\
3.8 \times 10^{6}\end{array}$ & $\begin{array}{l}0.1 \\
4.8 \\
1 \\
0.005 \\
0.4 \\
1.12 \\
201 \times 92 \\
3.2 \times 10^{6}\end{array}$ & $\begin{array}{l}0.1 \\
4.8 \\
0.5 \\
0.005 \\
0.2 \\
1.10 \\
401 \times 92 \\
1.9 \times 10^{6}\end{array}$ & $\begin{array}{l}0.1 \\
4.8 \\
0.5 \\
0.005 \\
0.2 \\
1.19 \\
401 \times 92 \\
2.1 \times 10^{6}\end{array}$ \\
\hline
\end{tabular}

replaced in the dissipative terms, excepting those with mixed spatial derivatives, by an effective value

$$
\tau_{\text {eff }}=\tau+\beta \rho h a,
$$

where $a$ is the velocity of sound. The parameter $\beta$ is given the smallest possible value that ensures the stability of the solution. This procedure is equivalent to introducing the effective viscosity into the QGD or QGDR equations in the $x, y$ formulation (Elizarova et al. 1995). The modified equations in axisymmetric coordinates have been given elsewhere (Lengrand et al. 1995).

The finite-difference equations are solved by means of an explicit algorithm where the steady-state solution is attained as the limit of a time-evolving process. The choice of time step is based on the stability condition in the form

$$
\Delta t=\alpha \min (h / a) .
$$

The computation finishes when the steady-state solution is reached according to the criterion

$$
\varepsilon=\frac{1}{N_{r} N_{z}} \sum_{h}\left|\frac{\rho^{j+1}-\rho^{j}}{\rho^{j} \Delta t}\right| \leqslant 10^{-7},
$$

where $j$ is the time-step index. Other details of the computational algorithm can be found elsewhere (Lengrand et al. 1995).

The convergence process to reach the steady-state solution is as follows: At the initial time $(t=0)$ the flow is regarded as an undisturbed cylindrical jet with radius $R_{e}$ characterized by $p_{e}, T_{e}, u_{z e}=u_{e}$ given in table 1 , with $u_{r}=0$, and undisturbed stationary $\left(u_{z}=u_{r}=0\right)$ gas around it with pressure $p=p_{\infty}$ and temperature $T=T_{\infty}$. At first the Mach disk defined by the condition $M a=1$ is formed at the distance $z \sim 120 R_{e}$ from the nozzle, then it moves towards the nozzle until $z \sim 50 R_{e}$, and only then it evolves away from the nozzle, slowly reaching its steady-state position at some point between $49 R_{e}$ and $55 R_{e}$, depending on the computational variant. This complex evolutional process determines the position of the downstream boundary of the computational domain at the distance equal to $200 R_{e}$.

The dependence of the results on the effective relaxation time, and on the grid, was studied by means of the three computational variants in table 2 , with the results shown in figure 3. Variants 1 and 2 of table 2 check the influence of the stabilizing parameter $\beta$ on the effective relaxation time. Increasing $\beta$ slightly smooths the gradients of all gasdynamic quantities $(\rho, p, T, u)$. Variants 1 and 3 in table 2 check the grid dependence of the results. Decreasing the spatial steps along $z$ (variant 3 ) leads to an insignificant increase of Mach numbers and shifts slightly the position of the Mach 


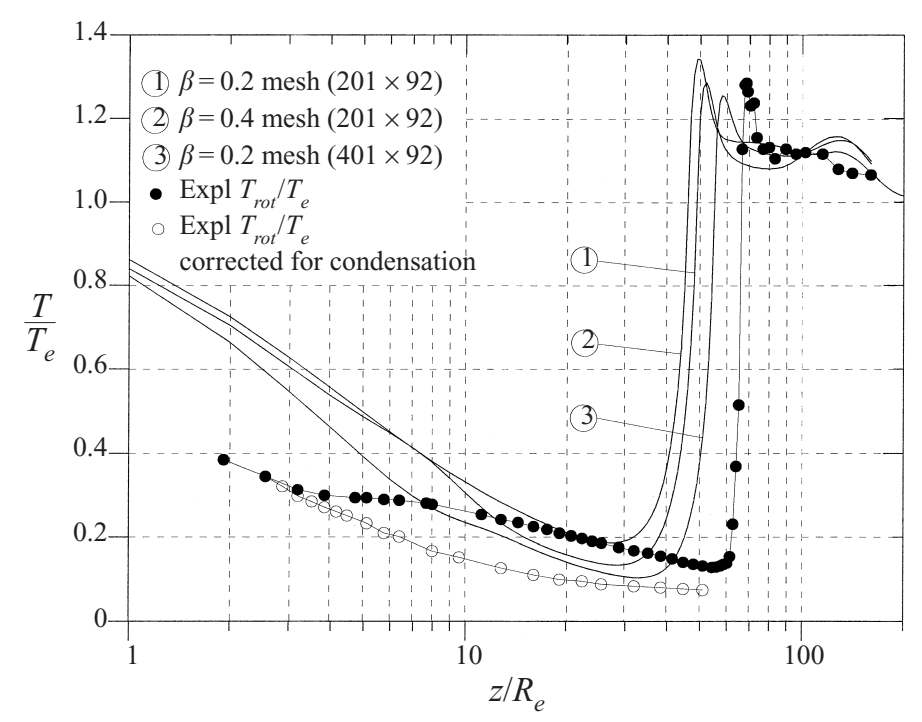

FIgURE 3. Temperature along the jet axis. Comparison of QGD variants 1, 2 and 3.

disk away from the nozzle by about one or two computational grid steps. In order to check the influence of the boundary layer on the flow properties two calculations were performed, one considering a boundary layer of $\delta=0.18 R_{e}$ at the nozzle exit, and the other neglecting the boundary layer $(\delta=0)$. At the very beginning of the expansion slightly higher temperatures $(\approx 1 \%)$ and densities $(\approx 4 \%)$ are obtained from the $\delta=0$ calculation, i.e. ignoring the boundary layer. This trend is reversed at the Mach disk, where $(\delta=0)$ temperatures are $\approx 6 \%$ lower and densities $\approx 1 \%$ higher than for a $\delta=0.18 R_{e}$ boundary layer. The position of the Mach disk is not affected, nor the remaining regions of the flow field.

\section{Results and discussion}

The flow investigated here is characterized by a strong non-uniformity. Density and pressure vary by several orders of magnitude in the axial direction, and by about one order of magnitude in the radial direction; temperatures vary by about one order of magnitude in each direction.

The spatial, axial, and radial distributions of flow quantities obtained by the QGD and QGDR methods are commented on next in comparison to experimental results. All quantities are plotted in dimensionless form, for the nozzle values quoted in table 1. Distances along and across the jet are referred to the nozzle radius $R_{e}=156.5 \mu \mathrm{m}$. The origin for $z$ was taken at the nozzle exit plane, and for $r$, at the symmetry axis. Note that all reported experimental temperatures are $T_{\text {rot }}$. Where appropriate, the isentropic prediction based on the empirical parametrization of $M a$ reported by Miller (1988) has been included for comparison.

\subsection{Axisymmetric flow field}

Density, temperature, and velocity maps presented in figure $1(a-d)$, summarize the properties of an axisymmetric supersonic jet. In $(a)$ and $(b)$ the experimental and QGDR calculated densities are compared. Due to the wide density range covered, only values of $n / n_{e}<10^{-3}$ are represented, corresponding approximately to distances 
$d>22 R_{e}$ from the nozzle. The general features of an axisymmetric supersonic jet, zone of silence, Mach disk, barrel shock, and slip region downstream of the Mach disk are evident, with a qualitative agreement of theory with experiment. Calculated and experimental barrel shock, position of Mach disk, and subsonic slip region are fairly similar, as are the quantitative values of $n / n_{e}$. There are however discrepancies in the details, mainly at the beginning of the expansion, and in the gradient and shape of the Mach disk. The former are likely to be related to the boundary conditions at the external nozzle wall, which are probably too idealized. Calculated Mach disk gradients are smoother than the experimental ones. Along the barrel shock the experimental densities are modulated periodically. The calculation seems to foreshadow this modulation. To confirm this point, a finer grid discretization should be employed along the radial direction in future calculations. Along the barrel shock, immediately after the Mach disk, a region of enhanced density evident in experimental and in calculated maps at $r / R_{e} \approx 20$ and $z / R_{e} \approx 75$, reveals the interference between barrel and normal shocks.

The calculated QGDR rotational temperature map on figure $1(d)$ is also in good qualitative agreement with its experimental counterpart, shown in figure $1(c)$. Along the barrel shock the calculated and experimental temperature distributions are similar. Beyond the Mach disk the barrel shock is elongated as a slightly converging channel of temperature lower than its surrounding environment. This feature, apparently related to the slip between the barrel shock and trapped vortex, is remarkably well reproduced by the QGDR calculation. The calculated thermal Mach disk is broader than the experimental one, as happens for density, and its curvature is reversed.

In the maps of figure $1(b, d)$, calculated velocities are superimposed onto densities and temperatures in order to visualize their correlation. In the zone of silence the flow field is nearly radial, the density and temperature decreasing with the distance to the nozzle. Approaching the barrel and normal shocks velocities become deflected, and density and temperature increase. At the barrel shock this deflection is smooth, with a slight reduction of velocity consistent with moderate rethermalization, and the tendency of the flow to evolve parallel to the symmetry axis. At the external boundary of the barrel shock the velocity remains close to the nozzle exit value $v_{e}=259 \mathrm{~m} \mathrm{~s}^{-1}$, but within the barrel shock the flow is supersonic. This combination of high density and high velocity shows that the distribution of momentum associated with the supersonic jet is largely confined at the barrel shock.

Unlike the barrel shock, at the Mach disk the flow is abruptly deflected outwards from the symmetry axis, with a strong reduction of velocity and transfer of kinetic energy into thermal energy. According to the calculation, this deflection coincides with the formation of a trapped vortex beyond the Mach disk, marked $(x)$ in figure $1(b, d)$, with a recirculation zone associated with a slow toroidal flow similar to that described recently (Chen, Chakravarthy \& Hung 1994; Stenholm \& Jover 1997; Welsh 1997; Gribben et al. 1998; Frey \& Hagemann 1998). In this structure the centreline velocity is reversed with respect to that in the zone of silence, differing qualitatively from the post-shock behaviour in one dimension. The toroidal trapped vortex appears to be responsible for the collimation of the jet downstream from the shock wave. However, at the present state of research it cannot be excluded that the vortex might be of computational origin as a consequence of the $(r, z)$ problem formulation, where screwlike three-dimensional flows are not possible.

To complete the information about two-dimensional flow-field properties, QGDR calculated iso-Mach lines depicted in figure 4 show the subsonic-supersonic boundaries of the jet. 


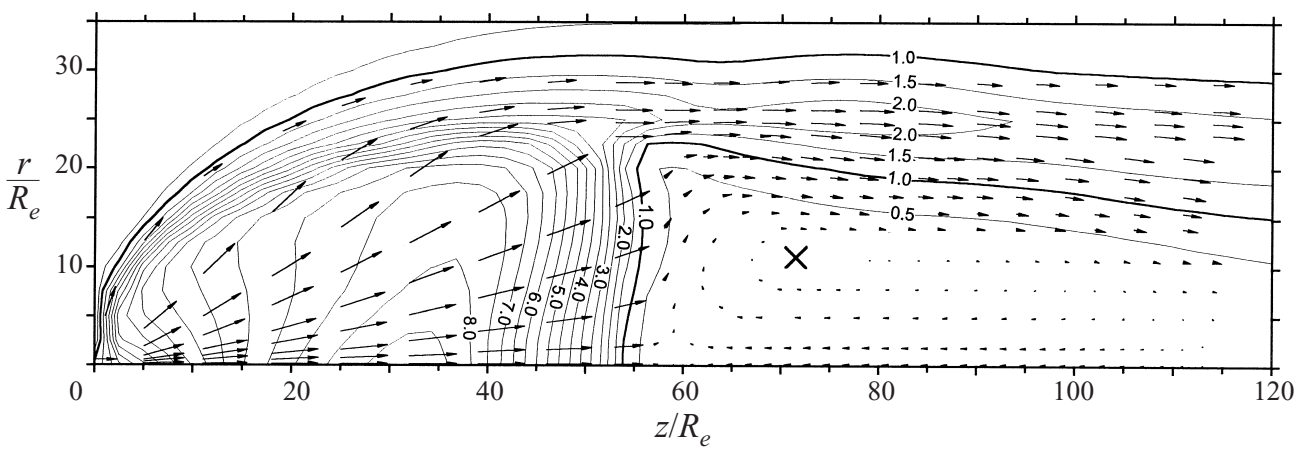

FIGURE 4. QGDR calculated iso-Mach lines and velocities in the supersonic jet of $\mathrm{CO}_{2}$. Source and nozzle exit conditions are given in table 1 .

\subsection{Axial and radial sections of the flow}

For a quantitative discussion of the jet in terms of the QGD and QGDR computational variants of table 2 , a selection of axial and radial sections is presented in figures 3 , and 5 to 10 .

\subsubsection{Temperature}

QGD axial temperature distribution obtained from variants 1, 2, and 3 of table 2 are presented in figure 3, intended to show the effect of mesh discretization, and of stabilizing parameter $\beta$, on the calculated temperature. Variants 1 and 3 of the calculation show how the present QGD results are affected by finite discretization along the jet axis: doubling the number of mesh points along $z$ reduces the error in positioning of thermal Mach disk from $24 \%$ to $12 \%$ respect to the experimental location. Variant 2 shows the strong influence of the stabilizing parameter $\beta$, resulting in smoothing of the gradients with increasingly large values of $\beta$ by moving upstream the minimum and the maximum of the axial temperature distribution; the value $\beta=0.1$ is too small, leading to an unstable solution. So, to show the influence of $\beta$ the flow field has been recalculated with $\beta=0.4$. Experimental rotational temperature, as measured, and condensation-corrected rotational temperature (Maté et al. 1998) are included in figure 3 for comparison.

QGDR on-axis rotational temperature is shown in graph 1 of figure 5, to be compared with the rotational experimental temperature corrected for condensation (Maté et al. 1998). For $z / R_{e}<10$ the calculation overestimates $T_{\text {rot }}$, but for $15<$ $z / R_{e}<40$ the agreement is excellent. The axial QGD temperature profile is close to the QGDR $T_{a v}$ profile. The $\gamma=1.35$ and $\gamma=1.40$ isentropic temperatures are included in figure 5 for comparison.

Graph 2 of figure 5 plots the ratio $T_{r o t} / T_{t r}$ of rotational to translational temperature, showing the breakdown of rotational-translational equilibrium predicted by the QGDR calculation. The rotational-translational equilibrium is largely maintained along the zone of silence, up to the shock wave. There the slight increase of rotational temperature $T_{r o t} / T_{t r} \simeq 1.03$ observed at $z / R_{e} \simeq 30$ is qualitatively consistent with the difference $\delta=T_{r o t}-T_{t r} \approx 4 \mathrm{~K}$ reported for the same experimental conditions (Maté et al. 1998).

At the onset of the normal shock wave, at about $z / R_{e} \geqslant 40$, the rotationaltranslational equilibrium breaks. Two thermal overshoots are predicted, one for $T_{t r}$ at $z / R_{e} \approx 54$, and the other for $T_{r o t}$ at $z / R_{e} \approx 60$. As shown in figure 5 the calculated 


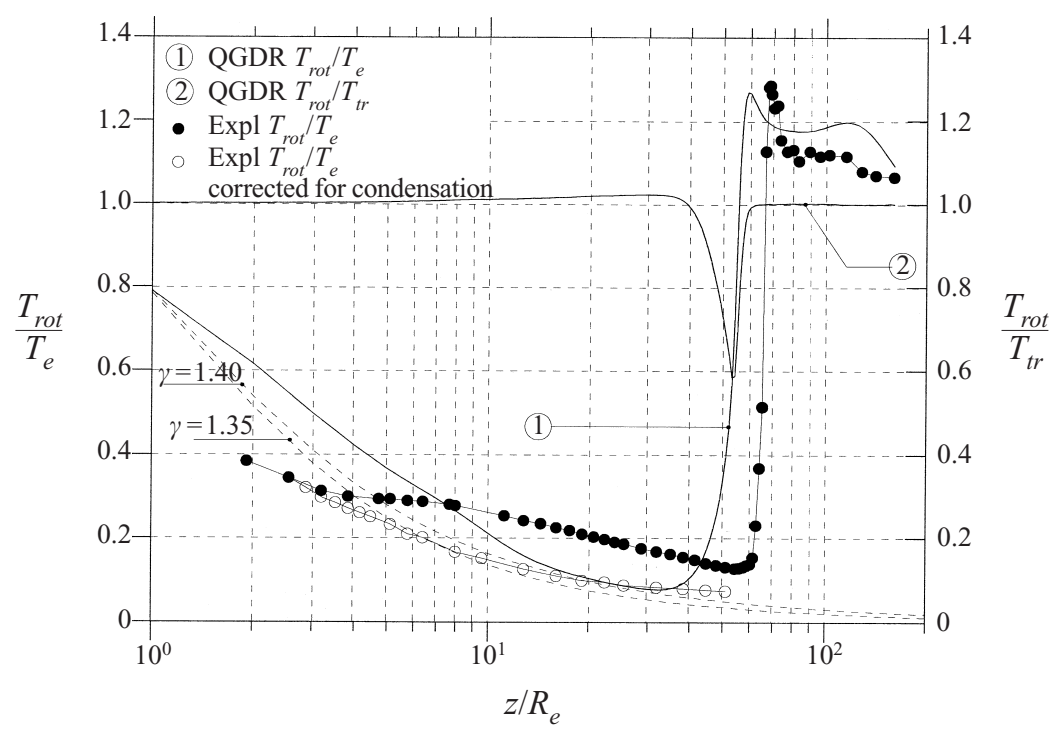

FIGURE 5. Temperature along the jet axis. Translational and rotational temperatures in QGDR calculations versus experiment, and isentropic temperature for $\gamma=1.35$ and $\gamma=1.40$.

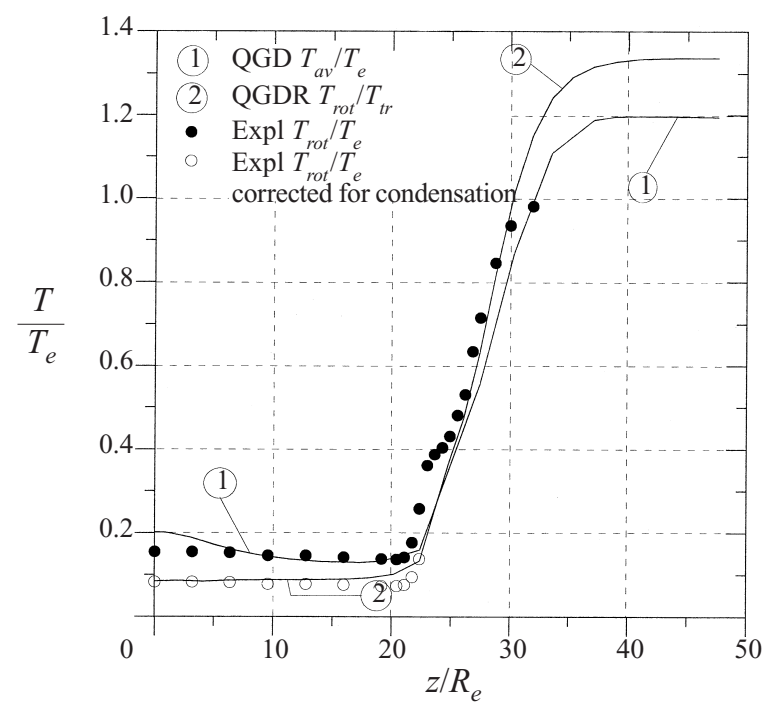

FIGURE 6. Temperature along the jet radius in the plane $z / R_{e}=38$. QGD and QGDR calculations versus experiment.

$T_{\text {rot }} / T_{e}$ overshoot, reaching a peak value of 1.27 , is in excellent agreement with experiment. The lowest calculated ratio, $T_{r o t} / T_{t r}=0.58$, reached at the point of the $T_{t r}$ overshoot, coinciding with the $M a=1$ supersonic to subsonic transition, indicates to what extent the rotational-translational equilibrium is broken. In this process the rotational collision number varies from $Z \sim 5$, at the nozzle exit, to $Z \sim 0.8$ in front of the Mach disk, and again to $Z \sim 5$ behind it.

Radial behaviour of temperatures upstream from the Mach disk, at the plane $z / R_{e}=38$, is shown in figure 6. Here the agreement of the QGDR calculation with experiment proves excellent. The flat tendency of the zone of silence along the radial 


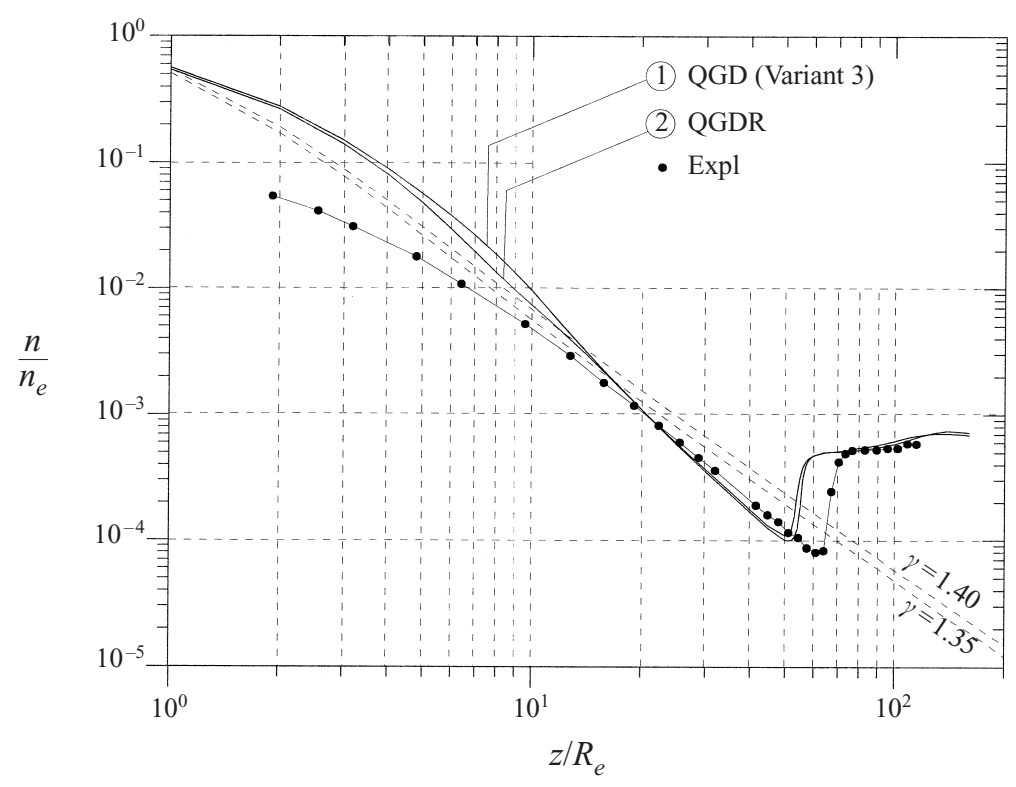

FIGURE 7. Density along the jet axis. QGD and QGDR calculations versus experiment, and isentropic density for $\gamma=1.35$ and $\gamma=1.40$.

direction, up to approximately $r / R_{e}<22 R_{e}$, is well described by the QGDR, but not by the QGD calculation. The slight bump, with slope change in the experimental data at the beginning of the barrel shock, in the range $23<r / R_{e}<26$, is due to the breakdown of the Boltzmann distribution of population of the rotational levels of $\mathrm{CO}_{2}$. This feature cannot be accounted for by the present QGD nor QGDR modelizations. In this range the experimental rotational temperatures reported in figure 6 correspond to best linear fits to Boltzmann plots. Unlike a normal shock wave, at the barrel shock the QGDR calculation predicts just a mild breakdown of rotational-translational equilibrium.

In spite of the qualitative agreement between calculated and experimental temperatures, two quantitative discrepancies deserve mention: (i) axial temperatures are systematically overestimated by the calculation for $z / R_{e}<7$, and (ii) the onset of the Mach disk is smoother in the calculated flow field than in the experiment. The first discrepancy is attributed to the too idealized boundary conditions at the $\mathrm{BC}$ wall, near the nozzle (see figure 2); the second, to the oversimplified description employed here for $\mathrm{CO}_{2}$ viscosity as a function of the temperature in a region of very strong thermal gradient and high local Knudsen number $(K n=0.21)$, close to the limit of applicability of models based on a continuum (Bird 1994). This effect can still be aggravated by the effective dissipation, proportional to the coefficient $\beta$ introduced in the computation for stabilizing purpose.

\subsubsection{Density}

QGD and QGDR axial densities shown in figure 7 are fairly similar, proving that densities are nearly insensitive to the QGD and QGDR computational approaches. At the beginning of the expansion calculated densities are strongly overestimated with respect to experiment, as happens for the temperature. The slight density increment observed downstream of the Mach disk is correctly reproduced by the calculation. 


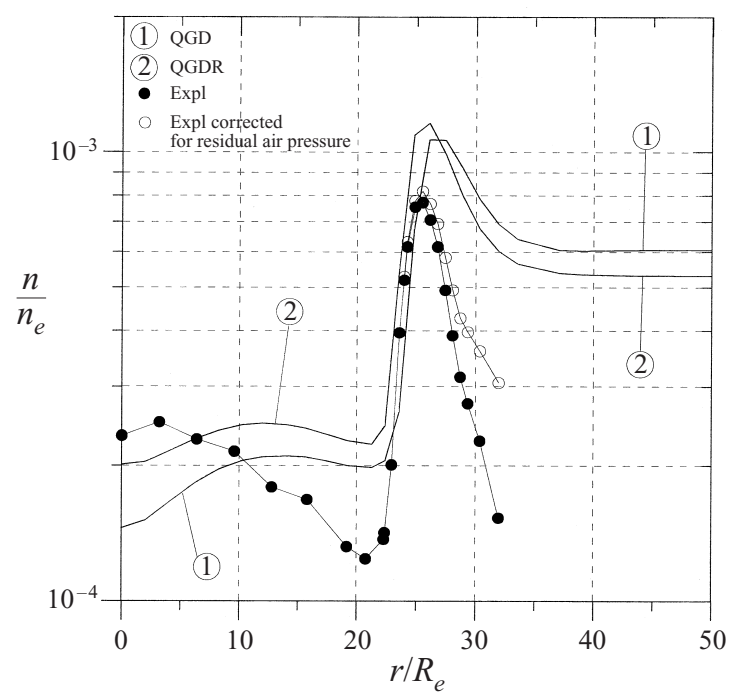

FIGURE 8. Density along the jet radius in the plane $z / R_{e}=38$.

QGD and QGDR calculations versus experiment.

Isentropic densities for $\gamma=1.35$ and $\gamma=1.40$, decaying asymptotically as $z^{-2}$, are shown in figure 7 as a reference.

With the criterion of maximum density gradient, equivalent to the $M a=1$ condition for all QGD and QGDR calculations performed here, the location of the density Mach disk is $z_{M}=49.5 R_{e}$ for variant $1, z_{M}=50.5 R_{e}$ for variant 2 , and $z_{M}=55.5 R_{e}$ for variant 3 of the QGD calculation, and $z_{M}=54.5 R_{e}$ for the QGDR calculation. The experimental value is $z_{M}=64 R_{e}$, and the empirical prediction according to (1) is $z_{M}=66 R_{e}$. Theoretical models neglecting viscous effects (Abramovich 1991) predict

$$
z_{M}=\left(2 R_{e}\right) \times 0.896 M a_{e} \sqrt{\gamma p_{e} / p_{\infty}} \approx 76 R_{e}
$$

for the nozzle conditions of table 1 , a $20 \%$ too large axial dimension for the zone of silence. From these values and trends one can conclude that the viscosity has been somewhat overestimated in the present modelization.

QGD, QGDR, and experimental radial densities in the plane $z / R_{e}=38$ are shown in figure 8. The QGDR calculated density jump and radial dimension of the density barrel shock agree well with experiment, but the calculated gradient beyond the barrel shock is smoother. This discrepancy is less pronounced if the experimental density is corrected for the contribution of air to the residual density, as described in $\S 2$. Finally, it is worth mentioning that according to experiment and QGDR calculation, at the barrel shock the density increment precedes the temperature increment, conversely to the normal shock wave.

\subsubsection{Pressure}

Pressure cannot be directly measured in the present experiments. However, it is possible to infer the rotational pressure from density and rotational temperature by means of the equation of state. Accordingly, the rotational pressure decreases along the expansion axis by about five orders of magnitude. The QGDR calculation, shown in figure 9, predicts a smaller decrease.

Across the normal shock wave the pressure increases, sharply according to ex- 


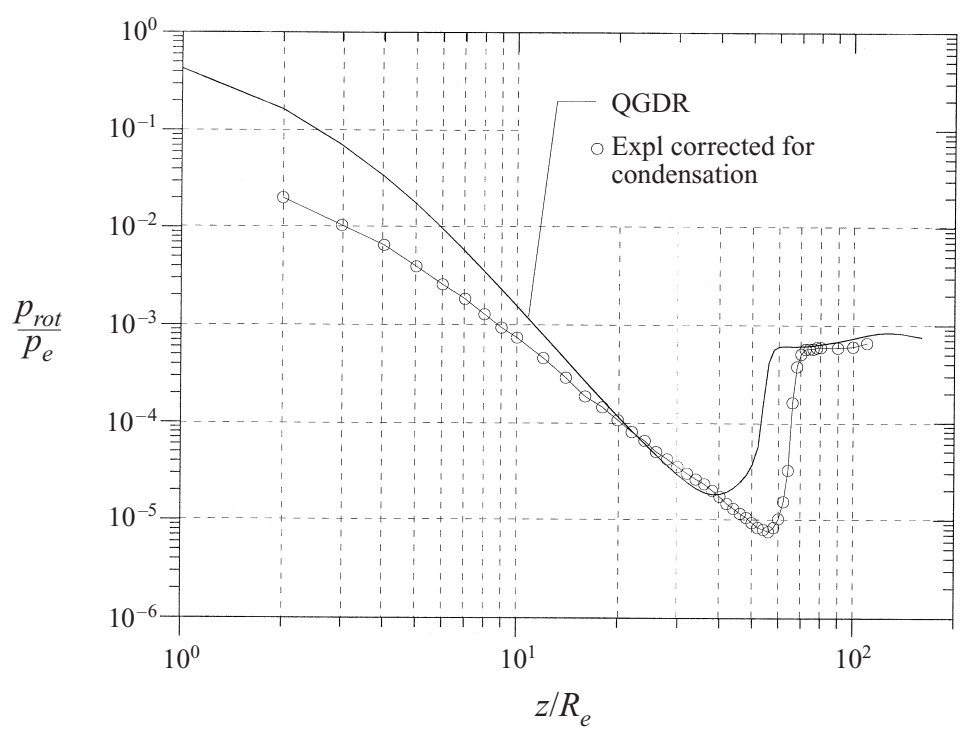

FIGURE 9. Pressure along the jet axis. QGDR calculation versus experiment (see text).

periment, and somewhat smoother according to calculation. This increase of pressure across the Mach disk by almost two orders of magnitude corresponds to the supersonic-subsonic transition in the jet. There is no evidence of secondary expansion beyond the Mach disk. On the contrary, the pressure remains close to the residual one, but with a slight positive pressure gradient along $z$, which is to be related to the inversion of velocities associated with the trapped vortex shown in figure $1(b, d)$. One may think of the positive pressure gradient as the driving mechanism of recirculation.

\subsubsection{Velocity and Mach number}

QGD and QGDR axial velocities are fairly similar in the zone of silence, with maximum values in the range $2.22<u_{z} / u_{z e}<2.37$, depending on computational variant. At the Mach disk $(M a=1)$ a strong deceleration is predicted and, as shown in figure 10 , at $z \approx 59 R_{e}$ the sign of the flow velocity becomes reversed. In the approximate range $59 R_{e}<z<116 R_{e}$ velocity is negative, in correlation with the positive pressure QGD and QGDR gradients shown in figure 9. The pressure gradient inferred from experiment, though positive, is far smoother, suggesting that the negative velocities are likely to be real but substantially smaller than predicted by QGD and QGDR calculations. Negative velocities, characteristic of two-dimensional calculations in the $r, z$ axisymmetric domain, are not expected in one-dimensional calculations. The inversion reported here is attributed to the trapped vortex discussed above. Note also that mass transfer $(\rho u)$ in this zone of the axis is sufficiently weak, approximately $2 \times 10^{-4}$ times less than near the nozzle. As may be deduced from figure $1(b)$ mass is mainly transferred along the barrel shock.

\section{Conclusions}

The scope of jet diagnostics using quantitative methods based on Raman spectroscopy is shown in present work in connection with the validation of numerical 


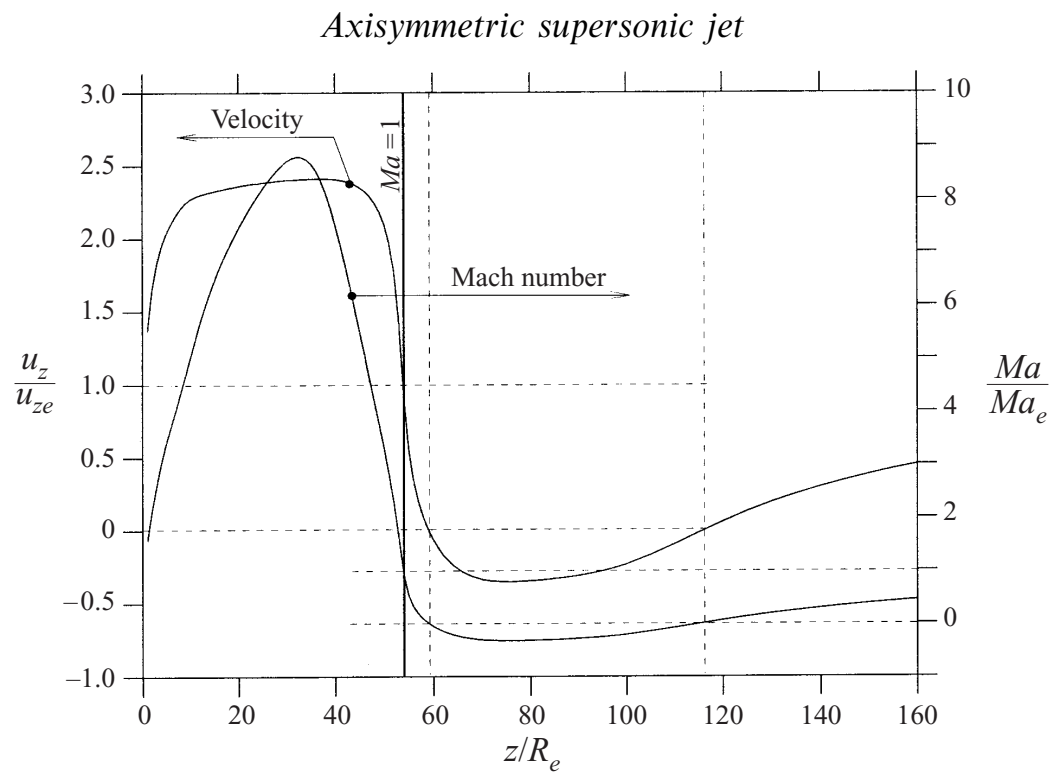

FIGURE 10. QGDR velocity and Mach number along the jet axis.

procedures in computational fluid dynamics. For that purpose the main physical quantities of an axisymmetric supersonic jet of $\mathrm{CO}_{2}$ were calculated using the QGD and QGDR equations, and were compared with experiment.

QGD and QGDR models do reproduce reasonably well the general structure of the jet, despite the large variation of Knudsen number from $K n_{e}=7.4 \times 10^{-5}$ at the nozzle exit, to a local value $K n=0.21$ within the Mach disk. There are however some specific quantitative differences with experiment. The calculated axial dimension of the zone of silence of the jet is about $15 \%$ too short. The radial dimension is reproduced well, especially by the QGDR approach.

For distances within $10 R_{e}$ from the nozzle, calculated densities and temperatures are systematically overestimated with respect to experiment. This is attributed to an oversimplification of the boundary conditions at the nozzle. On the other hand the excessive smoothness of temperature gradients at the onset of the Mach disk are attributed to intrinsic limitations in the modelling of $\mathrm{CO}_{2}$ viscosity as a function of local temperature. In any case, the results of QGD and QGDR calculations are good enough to give some credibility to those calculated quantities less amenable to experimental observation. Such is the case for velocities and Mach numbers, especially in connection with the trapped vortex downstream from the Mach disk.

The superiority of the QGDR over QGD model is evidenced in those regions with marked breakdown of rotational-translational equilibrium, like the rethermalization region of the Mach disk, where the calculated QGDR rotational temperature profile shows a better agreement with experiment than the QGD profile.

The present results suggest the necessity of more systematic work in order to investigate the influence of the boundary conditions at the nozzle, the role of the boundary layer, the breakdown of thermal equilibrium between longitudinal and transversal translational degrees of freedom, and the functional dependence of gas viscosity on temperature. As far as the experimental aspects of the present work are concerned, it may be concluded that Raman spectroscopy provides a useful tool for quantitative diagnostics of jets and related gasdynamic structures by virtue of its high throughput, excellent spatial resolution, accuracy in the measurement of rotational 
temperatures, and wide range of linearity in the measurement of densities. These capabilities can be further improved and extended to other quantities.

It is hoped that the interactive cooperation between experimental and numerical methods, along the lines initiated with present work, will lead to significant progress in the understanding of supersonic gasdynamic problems.

This research was supported by the Spanish Dirección General de Investigación Científica y Enseñanza Superior (DGICYES), Research Projects PB94-1526 and PB97-1203, and by the Fund for Fundamental Investigations of the Russian Academy of Sciences N 98-01-00155. The contribution of Yu. Sheretov to the $(r, z)$ formulation of the QGD equations and to the correction procedure is gratefully acknowledged.

\section{REFERENCES}

Abramovich, G. N. 1991 Applied Gas Dynamics, Vol. 1. Nauka, Moscow (in Russian).

AlsMeYer, H. 1976 Density profiles in argon and nitrogen shock waves measured by the absorption of an electron beam. J. Fluid Mech. 74, 497-513.

Ashrenas, H. \& Sherman, F. S. 1964 The structure and utilization of supersonic free jets in low density wind tunnels. In Proc. 4th Intl Symp. on Rarefied Gas Dynamics, Vol. 2, pp. 84-105.

Bier, K. \& SCHMidT, B. 1961 Zur Form der Verdichtungstöße in frei expandierenden Gasstrahlen. Z. Angew. Math. Phys. 13, 493-500.

Bird, G. A. 1994 Molecular Gas Dynamics and the Direct Simulation of Gas Flows. Clarendon.

CAmpargue, R. 1984 Progress in overexpanded supersonic jets and skimmed molecular beams in free-jet zones of silence. J. Phys. Chem. 88, 4466-4474.

Chapman, S. \& Cowling, T. 1952 The Mathematical Theory of Non-Uniform Gases. Cambridge University Press.

Chen, C. L., Chakravarthy, S. R. \& Hung, C. M. 1994 Numerical investigation of separated nozzle flows. AIAA J. 32, 1836-1843.

Chirokov, I. A., Elizarova, T. G. \& Lengrand, J. C. 1999 Numerical study of shock wave structure based on quasigasdynamic equations with rotational nonequilibrium. In Proc. 21st Intl Symp. on Rarefied Gas Dynamics (ed. R. Brun), Vol. 1, pp. 175-182. Cépaduès Editions.

Elizarova, T. G. \& Chetverushinin, B. N. 1985 Kinetic algorithms for calculating gasdynamic flows. J. Comput. Math. Phys. 25, 164-169.

Elizarova, T. G., Graur, I. A., Chpoun, A. \& Lengrand, J. C. 1997 Comparison of continuum and molecular approaches for the flow around a perpendicular disk. In Proc. 20th Intl Symp. on Shock Waves (ed. B. Sturtevant), pp. 795-800. World Scientific.

Elizarova, T. G., Graur, I. A., Lengrand, J. C. \& Chpoun, A. 1995 Rarefied gas flow simulation based on quasi-gas dynamic equations. AIAA J. 33, 2316-2324.

Elizarova, T. G., Lengrand, J. C. \& Graur, I. A. 1999 Gradient expansions for distribution functions and derivation of moment equations. In Proc. 21st Intl Symp. on Rarefied Gas Dynamics (ed. R. Brun), Vol. 1, pp. 119-126. Cépaduès Editions.

Frey, M. \& Hagemann, G. 1998 Status of flow separation prediction in rocket nozzles. AIAA Paper 98-3619.

Graur, I. A., Elizarova, T. G. \& Lengrand, J. C. 1997 Quasigasdynamic equations with multiple translational temperatures. Rep. R97-1. Laboratoire d'Aérothermique du CNRS, Meudon, France, ISSN 0248451X.

Graur, I. A., Elizarova, T. G. \& Lengrand, J. C. 1999 Numerical computation of shock wave configurations in underexpanded jets. Rep. R99-2. Laboratoire d'Aérothermique du CNRS, Meudon, France, ISSN 0248451X.

Gribben, B. J., Cantarini, F., Badcock, K. J. \& Richards, B. 1998 Numerical Study of an Underexpanded Jet. In Proc. Third Eur. Symp. on Aerothermodynamics for Space Vehicles. ESTEC. ESA SP-426, pp. 111-118.

Ivanov, M. S., Markelov, G. N., Gerasimov, Yu. I., Krylov A. N., Mishina, L.V. \& Sokolov, E. I. 1998 Free-flight experiment and numerical simulation for cold thruster plum. AIAA Paper 98-0898. 
Koura, K. 1997 Monte Carlo direct simulation of rotational relaxation of diatomic molecules using classical trajectory calculations: Nitrogen shock wave. Phys. Fluids 9, 3543-3549.

Lengrand, J. C., Chroun, A., Graur, I. A. \& Elizarova, T. G. 1995 Supersonic rarefied gas flow around a perpendicular disk. Rep. R95-6. Laboratoire d'Aérothermique du CNRS, Meudon, France, ISSN 0248451X.

Luijks, G., Stolte, S. \& Reuss, J. 1981 Molecular beam diagnostics by Raman scattering. Chem. Phys. 62, 217-229.

Maté, B. 1997 Characterization of supersonic expansions by Raman spectroscopy. Thesis (in Spanish). Universidad Autónoma de Madrid.

Maté, B., Tejeda, G. \& Montero, S. 1998 Raman spectroscopy of supersonic jets of $\mathrm{CO}_{2}$ : Density, condensation, and translational, rotational and vibrational temperature. J. Chem. Phys. 108, 2676-2685.

Miller, D. R. 1988 Free jet sources. In Atomic and Molecular Beam Methods (ed. G. Scoles), Vol. I, pp. 14-53. Oxford University Press.

Montero, S., Maté, B., Tejeda, G., Fernández, J. M. \& Ramos, A. 2000 Raman studies of free jet expansion. Diagnostics and mapping. In Atomic and Molecular Beams (ed. R. Campargue). Springer (in press).

Motт-Sмiтh, H. M. 1951 The solution of the Boltzmann equation for a shock wave. Phys. Rev. 82, 885-892.

Muntz, E. P., Hamel, B. B. \& Maguire, B. L. 1970 Some characteristics of exhaust plume rarefaction. AIAA J. 8, 1651-1658.

Murphy, H. R. \& Miller, D. R. 1984 Off axis properties of free-jets from different subsonic flows. In Proc. 14th Intl Symp. on Rarefied Gas Dynamics (ed. H. Oguchi), Vol. II, pp. 743-750. University of Tokyo Press.

Parker, J. G. 1959 Rotational and vibrational relaxation in diatomic gases. Phys. Fluids 2, 449-462.

Pham-Van-DieP, G., Erwin, D. \& Muntz, E. P. 1989 Nonequilibrium molecular motion in a hypersonic shock wave. Science 245, 624-626.

Robben, F. \& TALbot, L. 1966a Measurement of shock wave thickness by the electron beam fluorescence method. Phys. Fluids 9, 633-643.

Robben, F. \& Talbot, L. $1966 b$ Experimental study of the rotational distribution function of nitrogen in a shock wave. Phys. Fluids 9, 653-662.

Schlichting, G. 1955 Boundary Layer Theory. Pergamon.

SHERETOV, YU. V. 1997 Quasihydrodynamic equations as a model for viscous compressible heat conductive flows. In Implementation of Functional Analysis in the Theory of Approaches, pp. 127-155. Tver University (in Russian).

SHeretov, Yu. V. 1999 Hydrodynamic finite-difference schemes in the Euler and Lagrange coordinates based on quasigasdynamic and quasihydrodynamic equations. In Implementation of Functional Analysis in the Theory of Approaches, pp. 184-208. Tver University (in Russian).

Silvera, I. F. \& Tommasini, F. 1976 Intracavity Raman scattering from molecular beams: Direct determination of local properties in an expanding jet beam. Phys. Rev. Lett. 37, 136-139.

Stenholm, T. \& Jover, V. 1997 Flow separation control activities at Volvo and SEP. ESA Advanced Nozzle Workshop, University of Rome, Italy, October 1997.

Tejeda, G., Fernández-Sánchez, J. M. \& Montero, S. 1997 High-performance dual Raman spectrometer. Appl. Spectrosc. 51, 265-276.

Tejeda, G., Maté, B., Fernández-Sánchez, J. M. \& Montero, S. 1996 Temperature and density mapping of supersonic jet expansions using linear Raman spectroscopy. Phys. Rev. Lett. 76, 34-37.

WELSH, F. P. 1997 Electron beam fluorescence measurements of shock reflection hysteresis in an underexpanded jet. In 21st Intl Symp. on Shock Waves, Great Keppel, Australia, unpublished. 